\title{
Aquaculture Sentinels: Smart-farming with Biosensor Equipped Stock
}

\section{Andrewartha SJ1,2*, Elliott NG ${ }^{1,2}$, McCulloch $\mathrm{JW}^{3}$ and Frappell PB ${ }^{1,2}$}

${ }^{1}$ Integrated and Sustainable Aquaculture Production, CSIRO Agriculture, CSIRO Marine, Laboratories, Castray Esplanade, Hobart 7000, Australia ${ }^{2}$ Climate Change Physiology Laboratory, Institute for Marine and Antarctic Studies, University of Tasmania, 20 Castray Esplanade, Battery Point 7004, Australia

${ }^{3}$ Cyber Physical Systems Data61, CSIRO Marine Laboratories, Castray Esplanade, Hobart 7000, Australia

\begin{abstract}
Aquaculture is globally the fastest growing primary industry (>6\% per annum). Smart-farming, using sentinel animals equipped with miniature biosensors alongside environmental sensors and farm management systems has the potential to revolutionize all sectors of the industry. Real-time animal and environmental monitoring together, will support improved farm management decisions, animal welfare, social awareness and consequently sustainable productivity.

Biosensors that monitor the physiology and behavior of sentinel animals provide information on animal well-being and its responses to environmental change and management actions. In turn, this information is extrapolated to help with stock management decisions. This paper introduces the sentinel animal concept to commercial aquaculture with a case study using oysters fitted with biosensors that measure heart rate, and other parameters. We demonstrate how sentinel animals can be effectively integrated alongside environmental sensors into an on-farm sensor network and decision support system.
\end{abstract}

Keywords: Biologger; Biotag; Sensor network; Farm management; Smart farm; Decision support; Sentinel; Aquaculture; Heart rate; Valve activity

\section{Introduction}

Sentinel animals have been used for centuries. Perhaps the best known example is the miners' canary that served as an early warning signal for the buildup of toxic gases in the tunnels of coal mines. By definition, sentinels are individuals living within the common environment whose role is to provide early warning of sub-optimal conditions that may impact individual or population welfare and survival. Such conditions in commercial food production may be environmental, biological or due to management and the impacts will lead to less sustainable production and lower animal welfare. Today, we no longer need to wait for our sentinel animal to suffer or indeed expire. Instead, as with human health, we can monitor changes in an animal's physiology and/or behaviour using miniaturized electronics (biosensors), and capture and interpret data output in real-time using smart phones and other communication technologies. In brief, we can deliver more subtle and responsive early warning systems to support the end users; in this case aquaculture farmers.

Such information and communication technologies are already invaluable research tools for quantifying physiology, behaviour and large scale movement of free-ranging animals within their natural environment; though the data is often archived for later retrieval via satellite or following recapture [1]. In addition, biosensors are increasingly used to understand basic biology during experimental manipulation [2], and as biological early warning systems [3]. The usefulness of biosensors for informing agriculture management decisions is only just beginning to be realized [4], and here we introduce the concept of sentinel animals with biosensors as future core technology in aquaculture farm management.

\section{Seeing Through Water: Challenges in Monitoring Aquaculture Stock}

Aquaculture is a worldwide, multi-billion dollar industry ( USD 144.4 billion in 2012) with an average annual growth rate exceeding
$6 \%$ [5]. This industry will soon surpass wild seafood harvesting, and is essential to meet the growing global demand for protein and high nutritional diets $[5,6]$.

Aquaculture covers a range of animal species (teleosts, molluscs and crustaceans) as well as production systems (cages, ponds, open-sea racks, flow-through or recirculation) and environments (marine and freshwater; high and low intensity; temperate and tropical; subsistence farming to industrial scale production). However, all sectors share a common issue. Unlike terrestrial livestock farming, routine monitoring of the behaviour and health of thousands of individuals in a production system is extremely difficult. Visual monitoring of farmed aquatic animals and their responses to environmental change and management actions is not easy when animals are intensively reared underwater, or the animal is encased in a hard shell. Consequently, standard practice for traditional aquaculture monitoring involves the selection of random animals and then direct physical inspection of those individuals outside of their normal environment. A historic profile on an individual's or population's responses to environmental or management events is created via intermittent sub-sampling throughout production growout. The physical handling periods associated with these spot checks on welfare are themselves stress events, and any subsequent measurement or observation may be compromised [7]. Another common practice is to utilise easily-measured, environmental parameters such as water temperature and quality ( $\mathrm{pH}$, oxygen level, nitrogen, turbidity) as a proxy for animal well-being $[8,9]$. Recent developments in integrated

*Corresponding author: Sarah J Andrewartha, Integrated and Sustainable Aquaculture Production, CSIRO Agriculture, CSIRO Marine, Laboratories, Castray Esplanade, Hobart, Australia 7000, Tel: +613 62325208 E-mail: sarah.andrewartha@csiro.au

Received October 15, 2015; Accepted October 19, 2015; Published January 15 2016

Citation: Andrewartha SJ, Elliott NG, McCulloch JW, Frappell PB (2015) Aquaculture Sentinels: Smart-farming with Biosensor Equipped Stock. J Aquac Res Development 6: 393. doi:10.4172/2155-9546.1000393

Copyright: @ 2015 Andrewartha SJ, et al. This is an open-access article distributed under the terms of the Creative Commons Attribution License, which permits unrestricted use, distribution, and reproduction in any medium, provided the original author and source are credited. 
sensor networks have greatly improved the response time and reliability of environmental sensor technologies. Key environmental variables can be measured on farms in real-time and monitored remotely via a computer interface [10-12]. Nonetheless, while environmental sensors are often relatively cheap and provide valuable insight into stock living conditions they require regular cleaning and calibration, and importantly they do not provide any information concerning an animal's view of its environment, behaviour or physiology which are key indicators of well-being.

Understanding how their animals respond to environmental changes should be of essential interest to commercial farmers, both in the immediate production cycle and with the increasing unpredictability of climate change [13]. The development of small biosensors has enabled long-term, non-invasive monitoring of a range of physiological and/or behavioural variables that are either directly or indirectly relevant to animal health and productivity including heart rate, body temperature, movement, mollusc valve activity, and depth [14]. Sentinel animals fitted with biosensors that feedback to sensor networks have been developed for the dairy industry [4] and are currently being developed for farmed fish ([15] and CSIRO/UTAS unpublished) and commercially farmed molluscs (e.g. Pacific Oyster Case Study below). Real time physiological and behavioural data that provides insight into animal well-being has the ability to inform management practices and optimize production processes. Information about the environment is often required to interpret the physio-behavioural responses and the sentinel animal becomes integrated into an array of environmental sensors.

\section{Determining Animal Well-being and Productivity with Biosensors}

Biosensors can be designed to collect a broad variety of physiological and behavioural variables that are relevant to an individual's welfare and a population's productivity. Body temperature, animal orientation and depth have been measured directly in both wild and farmed fish [16]. Accelerometers used to quantify movement and in combination with pressure sensors and/or temperature sensors can characterize movement and feeding behaviour [17].

Changes in the normal physiology or behaviour patterns measured by biosensors may indicate stress or pathology particularly when associated with environmental change or known production stressors (Figure 1C). For example, biosensors have been used to determine pathology in humans and cattle by monitoring alterations in heart rate variability $[18,19]$.

Measured physiological variables can also be used to predict other important variables that are harder to measure on-farm. For example, feeding rates can be calculated from the characteristic increase in heart rate that occurs during digestion [20]. Energy expenditure is commonly estimated from accelerometry and/or heart rate, the latter being inextricably linked to aerobic metabolism [21]. Regardless of biosensor type, estimating energy expenditure is a powerful tool for the aquaculture industry because it enables growth rates to be predicted on-farm [22,23]. Rigorous laboratory calibration is required to establish the relationship between the measured variable (accelerometry or heart rate) and metabolic rate. This calibration is necessary for all environmental (e.g. temperature, oxygen, salinity) and farm "stressor" (e.g. food availability, disease, handling) conditions that the animals may encounter. Understanding how energy expenditure adjusts to management actions and environmental changes allows growth rates to be predicted for the often dynamically changing on-farm conditions.
Aquaculture industries are encouraged to embrace and utilize both environmental sensors and sentinel animals equipped with biosensors. Through these integrated sensor networks it will be possible to monitor the well-being and growth of aquaculture species and inform management decisions and practices, such as optimizing harvest timing, treating pathologies and, if required, delaying stressful activities such as size grading or transport.

\section{Pacific Oyster Case Study}

The Pacific oyster (Crassostrea gigas) is a global product, grown under a wide range of environments and subjected to a broad variety of production stressors. Oysters are sedentary and theoretically easy to monitor, however, their thick shell prevents close direct scrutiny and provides a challenge for bio-monitoring. In Australia, current commercial stock monitoring and evaluation occurs every 2.5-3 months when animals are collected from their grow-out environment for mechanical size grading $[24,25]$. Mortality and growth can be relatively easily assessed during this husbandry process, but it is highly labour intensive and thus, a major production expense. Therefore grading is not undertaken frequently, and does not provide knowledge on animal welfare or provide pre-warning on health issues. Further, prolonged air exposure and mechanical disturbance during grading can impact oyster wellbeing, causing an increase in stress markers such as circulating noradrenaline and dopamine, and reducing growth and survival rates [26,27]. Real-time data from in-situ sentinel animals with biosensors would reduce such stress and provide data to assist farm management decisions.

The need for bio-monitoring in shellfish such as oysters is also driven by regulator and consumer need for quality assurance. Costly and unpredictable local industry harvesting and sales closures result from oysters accumulating contaminants. Prediction of closures events, and duration, using sentinel animal monitoring has the potential to both reduce production losses and increase consumer confidence in the product. Networks of real-time environmental sensors (e.g.
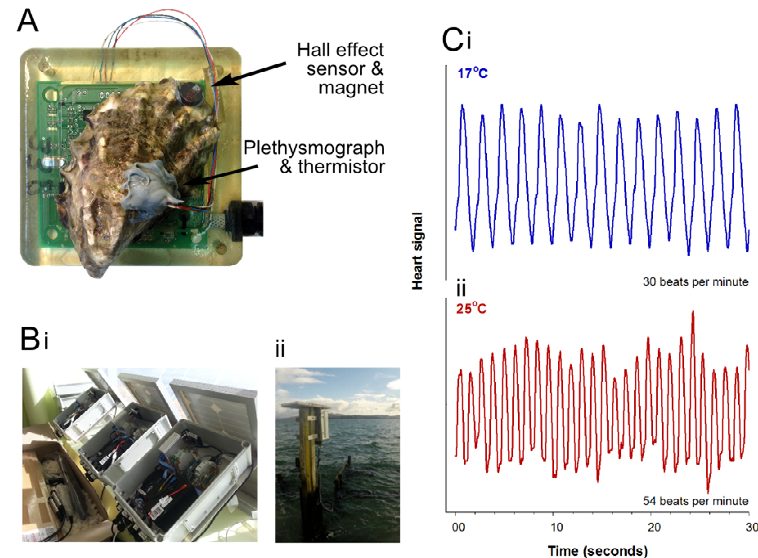

Figure 1: (A) Anoyster attached to UTAS/CSIRO oystag biosensor.The LED plethysmograph and thermistor are inserted through a small ( $<4 \mathrm{~mm}$ diameter) hole in the shell. A magnet attached to the top shell and the hall effect sensor on the printed circuit board (PCB) beneath are used to determine valve activity (i.e. shell gape). Additional sensors on the PCB are pressure (to inform depth), lux (light intensity) and ambient temperature. (Bi) The AgISP telemetry node and environmental sensors during preparation for installation and in the field. (Bii) The node transmits data from the oystag and environmental sensors to a data cloud via $4 \mathrm{G}$ technology.

(C) A representative oyster heart rate trace from the data cloud showing an increase in heart rate from $30 \mathrm{BP}$ Mat $17^{\circ} \mathrm{C}$ (i) to $54 \mathrm{BPM}$ at $25^{\circ} \mathrm{C}$ (ii). 
Citation: Andrewartha SJ, Elliott NG, McCulloch JW, Frappell PB (2015) Aquaculture Sentinels: Smart-farming with Biosensor Equipped Stock. J Aquac Res Development 6: 393. doi:10.4172/2155-9546.1000393

Page 3 of 4

salinity, temperature) deployed in catchments already feed into decision support models to aid regulators [28,29]. Our concept and research is to incorporate real-time data from oyster sentinels equipped with biosensors with environmental data to further improve decision support models by providing direct insight into animal wellbeing.

We have extended previously available technologies that monitored mollusc valve and cardiac activity in the short-term (48 h) [2] to longer term deployments within commercial grow out environments. Our oyster biosensors (Figure 1) not only monitor these two key parameters but also body temperature, water depth and light levels over time periods of weeks to months enabling data to be collected on-farm and processed in real-time.

Before the on-farm data can be interpreted it is critical to establish the important biological/environmental relationships through laboratory experiments under controlled environmental conditions; for example, heart rate and its correlation to metabolic rate across a range of water temperatures, and/or shell gaping as an indication of feeding. These relationships are determined across the known and predicted ranges of relevant environmental conditions and farm stressor events. Although time-consuming, this process in effect calibrates the sentinel biosensored animals so that field data can be interpreted and predictions about animal health and wellbeing can be made [21].

With this understanding, oysters fitted with biosensors have been deployed on farms alongside an appropriate array of environmental sensors that detect variables relevant to oyster health e.g. dissolved oxygen, salinity, $\mathrm{pH}$, chlorophyll $a$ concentration. The data is collected in real-time via a data cloud using $4 \mathrm{G}$ technology and can be accessed remotely (Figure 1). Oysters and their surrounding environment have been successfully monitored for up to 6 months. Changes in physiology, for example temperature dependent heart rate changes, are now routinely being monitored remotely (Figure 1C). Further field and laboratory ground truth data collection is on- going to ultimately establish an oyster well-being model to inform decision support systems around production (e.g. growth and maturation) and various stressors. For example, the farm decision support system may suggest that profits will be maximized by delaying a stressful farm activity such as mechanical grading because the oyster physiology shows they are still recovering from a recent high temperature exposure.

\section{Conclusion}

Smart farming offers oyster growers and other aquaculture farmers' access to unprecedented, timely and detailed information about their stock. Real-time physiological and behavioural cues from sentinel animals will provide indicators that enable swift management actions that prevent stock and consequently profit losses. Using sentinel animals to improve decision support systems prevent stock loss and increase yield can help the overall sustainability and profitability of oyster and other aquaculture industries.

\section{Acknowledgement}

The Pacific oyster case study was undertaken as part of the Sense-T program. Sense-T is a partnership between the University of Tasmania, CSIRO and the Tasmanian Government. It is also funded by the Australian Government. We also acknowledge Chris Sharman and Daniel Hugo for their technical contributions with the CSIRO Sensor Cloud technology, Brian Taylor for Oystag design and support and Justin Goc at Barilla Bay Oysters for facilitating on-farm data collection.

\section{References}

1. Hawkes LA, Balachandran S, Batbayar N, Butler PJ, Frappell PB, et al. (2011)
The trans-Himalayan flights of bar-headed geese (Anser indicus). Proc Nat Acad Sci U S A. 108: 9516-9519.

2. Curtis TM, Williamson R, Depledge MH (2000) Simultaneous, long-term monitoring of valve and cardiac activity in the blue mussel Mytilus edulis exposed to copper. Mar Biol 136: 837-846.

3. Kramer KM, Foekema E (2001) The "Musselmonitor®" as Biological Early Warning System. Biomonitors Biomarkers as Indic Environ Chang 2 56: 59-87.

4. Rutten CJ, Velthuis GJ, Steeneveld W, Hogeveen H (2013) Invited review: sensors to support health management on dairy farms. J Dairy Sci 96: 1928-52.

5. FAO Fisheries and Aquaculture Department (2014) The State of World Fisheries and Aquaculture - Opportunities and Challenges FAO, Rome.

6. Tacon AGJ, Metian M (2013) Fish Matters: Importance of Aquatic Foods in Human Nutrition and Global Food Supply. Rev Fish Sci 21: 22-38.

7. Segner H, Sundh H, Buchmann K, Douxfils J, Sundell KS, et al. (2012) Health of farmed fish: Its relation to fish welfare and its utility as welfare indicator. Fish Physiol. Biochem. 38: 85-105.

8. Schneider O, Schram E, Kals J, van der Heul J, Kankainen M, et al. (2012) Welfare Interventions in Flatfish Recirculation Aquaculture Systems and Their Economical Implications. Aquac Econ Manag 16: 399-413.

9. Simbeye DS, Zhao J, Yang S (2014) Design and deployment of wireless senso networks for aquaculture monitoring and control based on virtual instruments. Comput Electron Agric 102: 31-42.

10. Li Z, Zhang L, Li D, Fu Z (2012) Design and Implementation of Real-Time Monitoring and Intelligent Management System for Intensive Aquaculture. Sens Lett 10: 309-316.

11. Corke P, Wark T, Jurdak R, Hu W, Valencia P, Moore D (2010) Environmenta wireless sensor networks. Proc. IEEE 98: 1903-1917.

12. Zhu X, Li D, He D, Wang J, Ma D (2010) A remote wireless system for water quality online monitoring in intensive fish culture. Comput Electron Agric 71: 3-9.

13. IPCC (2014). Climate Change 2014: Synthesis Report. Contribution of Working Groups I, II and III to the Fifth Assessment Report of the Intergovernmental Panel on Climate Change [Core writing Team, R.K. Pachauri and L.A. Meyer (eds.)]. Geneva, Switzerland.

14. Cooke SJ, Hinch SG, Wikelski M, Andrews RD, Kuchel LJ, et al. (2004) Biotelemetry: A mechanistic approach to ecology. Trends Ecol Evol 19: 334-343.

15. Staaks G, Baganz D, Jauernig O, Berlin D (2010) The FischFITMonitor - A New System for Monitoring Multiple Physiological and Behavioural Parameters in Fish. Proceedings of Measuring Behavior, Eindhoven, The Netherlands.

16. Drenner SM, Clark TD, Whitney CK, Martins EG, Cooke SJ, et al. (2012) A synthesis of tagging studies examining the behaviour and survival of anadromous salmonids in marine environments. PLoS One 7: 1-13.

17. Payne NL, Taylor MD, Watanabe YY, Semmens JM (2014) From physiology to physics: are we recognizing the flexibility of biologging tools? J Exp Biol 217 317-22.

18. Mohr E, Langbein J, Nürnberg G (2002) Heart rate variability: A noninvasive approach to measure stress in calves and cows. Physiol Behav 75: 251-259.

19. Thayer JF, Yamamoto SS, Brosschot JF (2010) The relationship of autonomic imbalance, heart rate variability and cardiovascular disease risk factors. Int $J$ Cardiol 141: 122-131.

20. Clark TD, Taylor BD, Seymour RS, Ellis D, Buchanan J, Fitzgibbon QP Frappell PB (2008) Moving with the beat: heart rate and visceral temperature of free-swimming and feeding bluefin tuna. Proc Biol Sci 275: 2841-2850.

21. Green JA (2011) The heart rate method for estimating metabolic rate: Review and recommendations. Comp Biochem Physiol A: Mol Integr Physiol 158: 287-304.

22. Careau V, Killen S (2014) Adding fuel to the "fire of life": energy budgets across levels of variation in ectotherms and endotherms. Integrative Organismal Biology, John Wiley \& Sons, Inc. USA.

23. Carey MP, Zimmerman CE (2014) Physiological and ecological effects of increasing temperature on fish production in lakes of Arctic Alaska. Ecol Evol 4: 1981-1993. 
Citation: Andrewartha SJ, Elliott NG, McCulloch JW, Frappell PB (2015) Aquaculture Sentinels: Smart-farming with Biosensor Equipped Stock. J Aquac Res Development 6: 393. doi:10.4172/2155-9546.1000393

24. Nash C, Rubio-zuazo AM (2012) Monitoring the canaries of our catchments. In 21st NSW Coastal Conference, Australia.

25. Rubio A (2009) Using an automated oyster grading machine for long term monitoring of oyster performance. Science and Innovation Awards for Young People in Agriculture, Fisheries and Forestry sponsored by the Department of Agriculture, Fisheries and Forestry (DAFF).

26. Qu Y, Li X, Yu Y, Vandepeer M, Babidge P, et al. (2009) The effect of different grading equipment on stress levels assessed by catecholamine measurements in Pacific oysters, Crassostrea gigas (Thunberg). Aquac Eng 40: 11-16.
27. Spencer BE, Edwards DB, Millican PF (1992) Growing hatchery-reared Pacific oysters (Crassostrea gigas Thunberg) to marketable size in trays observations on costed small-scale culture methods and rough-handling trials. Aquaculture 106: 261-274.

28. Shahriar MS, Rahman A, McCulloch J (2014) Predicting shellfish farm closures using time series classification for aquaculture decision support. Comput Electron Agric 102: 85-97.

29. Shahriar MS, D'Este C, Rahman A (2012) On detecting and predicting harmful algal blooms in coastal information systems. OCEANS, 2012 - Yeosu, Yeosu, South Korea. 